\title{
CONHECIMENTO E CONDUTA EM RELAÇÃO AS INJÚRIAS DENTÁRIAS TRAUMÁTICAS DE PROFESSORES DO ENSINO FUNDAMENTAL DE JOÃO PESSOA-PB, BRASIL
}

\author{
KNOWLEDGE AND ATTITUDES TOWARDS DENTAL TRAUMA OF \\ SCHOOL TEACHERS IN JOÃO PESSOA-PB, BRAZIL
}

\author{
Heloísa Helena Pinho VELOSO'; Jussara Marinho de MELO; Danielly Raizer de OLIVEIRA²; Orlando Aguirre GUEDES²; Andréa \\ Sarmento QUEIROGA ${ }^{3}$ \\ 1 - Departamento de Odontologia Restauradora, Faculdade de Odontologia, Universidade Federal da Paraíba - UFPB, João Pessoa, PB, Brasil. \\ 2 - Departamento de Endodontia, Faculdade de Odontologia, Universidade de Cuiabá - UNIC, Cuiabá, MT, Brasil. \\ 3 - Departamento de Morfologia, Faculdade de Odontologia, Universidade Federal da Paraíba - UFPB, João Pessoa, PB, Brasil.
}

\section{RESUMO}

Objetivo: Avaliar o conhecimento de professores do ensino fundamental sobre as condutas nos casos de injúrias dentárias traumáticas (IDTs) em escolares. Material e método: O estudo foi realizado em uma amostra de 172 professores do ensino fundamental em João Pessoa-PB, e que responderam a um questionário com perguntas sobre os conhecimentos a cerca das condutas relacionadas ao tratamento emergencial de IDTs. Resultados: Os participantes do estudo eram em sua grande maioria do gênero feminino $(79,6 \%)$ e tinham entre 20 e 40 anos $(49,4 \%)$. Sobre os procedimentos a tomar após um acidente com trauma da face, $78 \%$ disseram que examinariam a boca das crianças. Com relação a possibilidade de salvar um dente fraturado, $78,5 \%$ dos entrevistados responderam positivamente. Em casos de dentes avulsionados, verificou-se que $7,6 \%$ dos professores não se preocupariam com o dente, apenas com a criança, e somente $33,7 \%$ procurariam o dente. O tempo ideal para procurar o cirurgião-dentista foi relatado por $52,8 \%$ como sendo no máximo 30 minutos. Quanto ao meio de armazenamento, $8,1 \%$ transportariam o dente imerso em leite e 29,7\% em soro fisiológico. Conclusões: O nível de conhecimento dos professores foi insuficiente frente ao atendimento de urgência de traumatismos dentários, necessitando-se de capacitação com o intuito de minimizar as perdas dentárias.

Palavras-chave: Conhecimento; Emergências; Traumatismos dentários.

\section{INTRODUÇÃO}

O ambiente escolar é propício à ocorrência de injúrias dentárias traumáticas (IDTs), as quais constituem numa das principais causas de perdas de dentes anteriores. Este é um local que concentra grande quantidade de crianças interagindo socialmente, praticando esportes e atividades físicas, correndo e brincando, estando propícias a quedas e acidentes que podem resultar em ITD. Estudos corroboram esta afirmação, mostrando que a maior parte dos traumatismos na região bucal ocorre durante os primeiros 12 anos de vida, e a escola é o local de maior incidência ${ }^{1,2}$.

A perda de um dente anterior leva à dificuldade de manutenção da estética. Em muitos casos, o desconhecimento sobre o assunto, especialmente pelas pessoas que estão próximas aos acidentados, familiares e até mesmo profissionais da área da saúde, leva à perda não só da função e estética, mas também em severo dano psicológico, afetando diretamente a interação do acidentado com a sociedade ${ }^{3-9}$.

Devido à importância da manutenção do dente em função pelo maior tempo possível, o que é preconizado pela Associação Internacional de Traumatismos Dentários (IADT), faz-se necessário que os profissionais e pessoas presentes em situações de potencial risco a acidentes estejam preparadas para o atendimento de urgência, com vistas a melhora do prognóstico e, consequentemente, da qualidade de vida dos acometidos por este traumas ${ }^{10,11}$.

No ambiente escolar, o professor é o profissional responsável e que está presente no momento em que ocorre uma IDT. Assim, é o professor quem deve prestar o primeiro atendimento às crianças acidentadas. Para isso, o mesmo precisa conhecer as situações de emergência a que podem ser acometidas estas crianças, além de estar apto a realizar os procedimentos emergenciais cabíveis ${ }^{12,13}$, haja vista a necessidade de atendimento rápido para que os acidentados possam apresentar o melhor prognóstico possível, não só no que concerne à manutenção do dente traumatizado, como também para sua saúde, como a necessidade de vacina antitetânica ${ }^{10,14}$.

Entretanto, estudos realizados em diversas regiões do mundo têm demonstrado que os professores não apresentam conhecimento suficiente para o atendimento emergencial ou mesmo para fornecer orientações a respeito do que deve ser feito em casos de IDTS ${ }^{4-7,9,15-23}$. Este desconhecimento resulta, em diversas situações, na perda de dentes ou no agravamento das condições que poderiam ser evitadas com medidas simples, como por exemplo, o conhecimento do melhor meio de armazenamento para transporte de dentes avulsionados. 
Nesse sentido, este trabalho objetivou avaliar o conhecimento de professores das escolas municipais de ensino fundamental do município de João Pessoa-PB, Brasil, no que concerne às condutas emergencias frente as ITDs.

\section{MATERIAL E MÉTODOS}

Este estudo foi aprovado pelo Comitê de Ética em Pesquisa da Universidade Federal da Paraíba (Proc. \#0261).

Aplicou-se questionários que avaliaram os conhecimentos necessários e as condutas relacionadas ao tratamento emergencial de IDTs. Os questionários foram entregues, juntamente com o termo de consentimento livre e esclarecido, aos responsáveis por cada escola para serem distribuídos a todos os professores do ensino fundamental da rede pública de ensino do município de João Pessoa, PB, Brasil, que totalizavam 3880 indivíduos. Cento e setenta e dois professores concordaram em participar da pesquisa por meio da assinatura do termo de consentimento e preencheram corretamente os questionários.

Os dados dos questionários foram registrados em planilha e submetidos à análise estatística por meio do software SPSS (Statistical Package for Social Sciences) para Windows, versão 20.0, e analisados por meio de estatística descritiva e inferencial bivariada.

Para a escolha dos testes estatísticos o teste de Kolmogorov-Smirnov apontou para a normalidade de distribuição dos dados. Os procedimentos de inferência estatística foram realizados por meio do teste de aderência, Qui-Quadrado $\left(\chi^{2}\right)$ e cálculo do coeficiente $\mathrm{V}$ de Cramer. Para todos os testes foi considerado nível de significância de 5\%.

\section{RESULTADOS}

Dos 172 professores participantes da pesquisa, a maioria foi do gênero feminino (79,6\%), e estavam entre 20 e 40 anos (49,4\%).

Inicialmente, foi questionado aos professores se estes examinariam a boca das crianças se ocorresse um acidente com trauma na face. Obteve-se resposta positiva em $78 \%(p<0,001)$. Foi também questionado aos professores se, em caso de fratura do dente após o traumatismo, eles procurariam o fragmento. Obteve-se um resultado estatisticamente significante de 70,4\% dos profissionais respondendo afirmativamente $(p<0,001)$. A grande maioria dos professores $(78,5 \%)$ respondeu que é possível salvar um dente que foi fraturado após um traumatismo, resultado estatisticamente significante $(\mathrm{p}<0,001)$ (Tabela 1$)$.

Em relação à atitude dos professores em caso de dentes avulsionados, verificou-se que $44,2 \%$ entrariam em contato com o responsável da criança para que este decidisse que atitude tomar $(\mathrm{p}=0,12)$. Apenas $7,6 \%$ dos professores informaram que não se preocupariam com o dente e estariam preocupados somente com a condição de saúde da criança $(\mathrm{p}<0,001)$. Já $33,7 \%$ relataram que procurariam o dente $(\mathrm{p}<0,001)$, ao passo que $30,8 \%$ levariam a criança para atendimento de urgência $(\mathrm{p}<0,001)$ (Tabela 2).

Foi questionado qual seria o tempo ideal para procurar o cirurgião-dentista depois de acontecer uma avulsão dentária. Mais da metade dos professores $(52,8 \%)$ informaram que este tempo é de até 30 minutos, 28,2\% relataram ser de até 4 horas, e 19\% de até $24 \mathrm{~h}(\mathrm{p}<0,001)$.

Quanto à forma de transportar um dente avulsionado, o leite seria o meio de transporte utilizado por $8,1 \%$ dos professores $(\mathrm{p}<0,001)$, enrolado em algodão ou guardanapo por $48 \%(\mathrm{p}=0,59)$, $29,7 \%$ utilizariam o soro fisiológico $(\mathrm{p}<0,001), 5,8 \%$ deixariam na boca da criança $(\mathrm{p}<0,001), 4,7 \%$ deixariam em água $(\mathrm{p}<0,001)$, e $8,7 \%$ não utilizariam qualquer meio, mantendo em um recipiente vazio $(\mathrm{p}<0,001)$.

O que fariam caso constatassem que um dente que caiu estava sujo foi a pergunta seguinte. A resposta mais prevalente foi aquela que sugeriu que lavaria apenas com água (56\%), com diferença estatisticamente significante. Dos demais, 4,2\% dos professores informaram que lavariam com água e escovariam o dente, 22\% relataram que lavariam com água e sabão e 17,9\% informaram que não fariam nada.

Foi questionado aos professores se já presenciaram casos de traumatismos dentários envolvendo os seus alunos. Apenas $14,6 \%$ dos participantes informaram ter presenciado tal evento $(\mathrm{p}<0,001)$. Ainda nesta perspectiva, foi questionado o número de traumatismos dentários que presenciaram. A maioria dos professores não lembrou quantos eventos desta natureza $(71,7 \%)$. Entretanto, 10,8\% informaram que presenciaram apenas um traumatismo, $10 \%$ informaram ter presenciado 2 traumatismos, e 7,5\% relataram ter presenciado 3 ou mais traumatismos, com diferença estatística significante $(\mathrm{p}<0,001)$.

Tabela 1 - Atitudes dos professores frente a um trauma na face.

\begin{tabular}{|c|c|c|c|c|c|c|c|}
\hline \multirow{2}{*}{ Variáveis } & \multicolumn{2}{|l|}{$\operatorname{Sim}$} & \multicolumn{2}{|c|}{ Não } & \multicolumn{2}{|c|}{ Não soube } & \multirow{2}{*}{ Valor de $p$} \\
\hline & $\mathrm{n}$ & $\%$ & $\mathrm{n}$ & $\%$ & $\mathrm{n}$ & $\%$ & \\
\hline Realização de exame bucal em casos de traumas na face* & 131 & 78,0 & 37 & 22,0 & - & - & $p<0,001$ \\
\hline Procura de fragmento dentário em casos de fratura do mesmo** & 119 & 70,4 & 50 & 29,6 & - & - & $p<0,001$ \\
\hline Possibilidade de salvar um dente fraturado & 135 & 78,5 & 4 & 2,3 & 33 & 19,2 & $p<0,001$ \\
\hline
\end{tabular}

* Quatro professores não responderam esta questão. ** Três professores não responderam esta questão.

Tabela 2 - Atitude frente a um dente avulsionado.

\begin{tabular}{|c|c|c|c|c|c|}
\hline \multirow{2}{*}{ Variáveis } & \multicolumn{2}{|c|}{$\operatorname{Sim}$} & \multicolumn{2}{|l|}{ Não } & \multirow{2}{*}{ - Valor de $p$} \\
\hline & $n$ & $\%$ & $\mathrm{n}$ & $\%$ & \\
\hline Entraria em contato com o responsável & 76 & 44,2 & 96 & 55,8 & $p=0,12$ \\
\hline Se importaria apenas com a criança & 13 & 7,6 & 159 & 92,4 & $p<0,001$ \\
\hline Procuraria o dente & 58 & 33,7 & 114 & 66,3 & $p<0,001$ \\
\hline Levaria a criança para atendimento de urgência & 53 & 30,8 & 119 & 69,2 & $p<0,001$ \\
\hline
\end{tabular}


Nos casos em que se relatou ter presenciado um acidente com traumatismo dentário associado, foi questionado aos professores se existiu algum tipo de notificação na escola. A maior parte não soube informar se isto aconteceu (50\%), e 26,7\% informaram que houve notificação $(p=0,003)$ (Tabela 3). Foi solicitada a opinião dos professores sobre a necessidade de criação de um protocolo de atendimento em casos de acidentes com traumatismos dentários. Verificou-se uma prevalência significativa que apoiou a criação de um protocolo $(78,3 \%, \mathrm{p}<0,001)$. No entanto, esta opinião não diferiu em função da idade dos participantes $(p=0,67)$ (Tabela 3). Por fim, também foi questionada a opinião dos professores sobre a criação de um anexo com informações médicas na ficha dos alunos. A maioria respondeu afirmativamente a este questionamento $(89,6 \%)$, também não diferindo em função da idade dos professores $(\mathrm{p}=0,35)$ (Tabela 3$)$.

\section{DISCUSSÃO}

Para que sejam adotadas as condutas de urgência adequadas frente a IDT é necessário conhecer as prioridades da situação, uma vez que o melhor prognóstico para o elemento dentário é obtido quanto mais rápido tenha sido realizado o primeiro atendimento ${ }^{2,6,15}$. O tempo decorrido entre o acidente e o atendimento para que o paciente apresente um prognóstico favorável deve ser inferior a sessenta minutos, especialmente nos casos de avulsão dentária ${ }^{10,14}$.

Este estudo encontrou um elevado índice (78\%) de professores que examinariam a boca das crianças se ocorresse um trauma na face. Isto mostra que os professores têm consciência de que nem sempre uma lesão resultante de um traumatismo é visível com um simples olhar. Além disso, 70,4\% dos professores procurariam o pedaço do dente em casos de fratura, resultado este que vem corroborar com as condutas sugeridas pelas diretrizes da Associação Internacional de Traumatismos Dentários $(\text { IADT })^{11}$. Chamou atenção o fato de que 21,5\% dos professores responderam não ser possível ou não ter conhecimento sobre a possibilidade de se salvar um dente fraturado pós-traumatismo. Este fato mostra a necessidade de políticas educativas quanto as IDTs visando não só a prevenção, como também a capacitação de profissionais da educação no que concerne ao tema.

Com relação à avulsão dentária, as atitudes assumidas pelos professores adiariam medidas que deveriam ser tomadas com urgência a fim de melhorar o prognóstico do caso. A IADT ${ }^{10}$ orienta que o atendimento deve acontecer em no máximo $60 \mathrm{mi}-$ nutos para que se tenha excelentes condições de tratamento, a fim de se evitar a ocorrência de reabsorções radiculares.
O tempo de exposição do dente avulsionado ao meio ambiente apresenta significativa influência sobre a viabilidade das células do ligamento periodontal. Exposições superiores a 60 minutos causam a necrose destas células, o que impede a regeneração do periodonto. No presente estudo, 58\% dos professores informaram que o tempo ideal para procurar o cirurgião-dentista depois de acontecer o acidente seria de até 30 minutos. Outros estudos também apresentaram resultados semelhantes ${ }^{7,16,24}$. Chamou atenção o fato de que $47,2 \%$ dos professores responderam tempos para o atendimento inadequados, o que deve levar um alto índice de perdas dentárias pós-traumatismos.

Quanto ao meio de armazenamento, a literatura mostra que ao levar-se em conta as características de preservação do ligamento periodontal e a maior disponibilidade em ambientes onde ocorrem os traumas, os melhores meios de transporte para dentes avulsionados são o leite e a água de $\operatorname{coco}^{10,14,25,26}$. Entretanto, ficou evidente que os professores desconheciam a melhor maneira de transportar o dente avulsionado até o momento do reimplante, uma vez que apenas $8,7 \%$ transportariam o dente imerso em leite. Outros estudos também apresentaram resultados insatisfatórios em relação ao conhecimento do meio de transporte adequado ${ }^{7,16,24}$.

O dente sujo seria lavado em água corrente pela maioria dos entrevistados, o que está de acordo com as diretrizes propostas pela IADT ${ }^{10}$. Entretanto, $44 \%$ dos professores tomariam condutas inadequadas, como lavar o dente com água e sabão ou escovar a raiz, o que seria prejudicial à vitalidade das células do ligamento periodontal e traria prejuízos ao prognóstico do $\operatorname{caso}^{10,25}$.

Apenas 14,6\% dos participantes informaram ter presenciado algum tipo de IDT. Esse dado é semelhante aos encontrados em outro estudo, em que $77,1 \%$ dos professores avaliados nunca viram um caso de traumatismo na escola ${ }^{18}$.

Metade dos professores que presenciaram casos de avulsão não soube informar se houve algum tipo de notificação na escola.

Os próprios professores concordaram com a necessidade da criação de um protocolo de atendimento em casos de acidentes, mais especificamente quanto aos traumatismos dentários, para propiciar uma forma única e correta de intervir nestes casos, propiciando melhor prognóstico ${ }^{18}$. As diretrizes de instituições como a IADT ${ }^{10}$ poderiam ser utilizadas na determinação destes protocolos.

\section{CONCLUSÃO}

O conhecimento dos professores mostrou-se insuficiente para um atendimento emergencial adequado em casos de IDTs, propiciando a expectativa de prognósticos desfavoráveis em grande parte destes casos.

Tabela 3 - Importância da notificação, protocolo de atendimento de urgência e presença de informações clínicas na escola.

\begin{tabular}{|c|c|c|c|c|c|c|c|}
\hline \multirow{2}{*}{ Variáveis } & \multicolumn{2}{|l|}{ Sim } & \multicolumn{2}{|c|}{ Não } & \multicolumn{2}{|c|}{ Não sei } & \multirow{2}{*}{ Valor de $\mathrm{p}$} \\
\hline & $\mathrm{n}$ & $\%$ & $n$ & $\%$ & $\mathrm{n}$ & $\%$ & \\
\hline Existência de notificação na escola em casos de acidentes* & 24 & 26,7 & 21 & 23,3 & 45 & 50,0 & $p=0,003$ \\
\hline $\begin{array}{l}\text { Necessidade de criação de um protocolo de atendimento para casos de trauma- } \\
\text { tismos dentários nas escolas** }\end{array}$ & 119 & 78,3 & 16 & 10,5 & 17 & 11,2 & $p<0,001$ \\
\hline $\begin{array}{l}\text { Necessidade de criação de um anexo com informações médicas na ficha de } \\
\text { matrícula do aluno*** }\end{array}$ & 138 & 89,6 & 7 & 4,5 & 9 & 5,8 & $p<0,001$ \\
\hline
\end{tabular}

* Oitenta e dois professores não responderam esta questão.

${ }^{* *}$ Trinta professores não responderam esta questão.

***Dezoito professores não responderam esta questão. 


\section{REFERÊNCIAS}

01. Andersson L. Epidemiology of traumatic dental injuries. J Endod. 2013;39(3 Suppl):S2-5.

02. de Amorim Lde F, da Costa LR, Estrela C. Retrospective study of traumatic dental injuries in primary teeth in a Brazilian specialized pediatric practice. Dent Traumatol. 2011;27(5):368-73.

03. Rezende FM, Gaujac C, Rocha AC, Peres MP. A prospective study of dentoalveolar trauma at the Hospital das Clínicas, São Paulo University Medical School. Clinics. 2007;62(2):133-8.

04. Mohandas U, Chandan GD. Knowledge, attitude and practice in emergency management of dental injury among physical education teachers: a survey in Bangalore urban schools. J Indian Soc Pedod Prev Dent. 2009;27(4):242-8.

05. Mori GG, Turcio KH, Borro VP, Mariusso AM. Evaluation of the knowledge of tooth avulsion of school professionals from Adamantina, São Paulo, Brazil. Dent Traumatol. 2007;23(1):2-5.

06. Ramroop V, Wright D, Naidu R. Dental health knowledge and attitudes of primary school teachers toward developing dental health education. West Indian Med J. 2011;60(5):576-80.

07. Raoof M, Zaherara F, Shokouhinejad N, Mohammadalizadeh S. Elementary school staff knowledge and attitude with regard to first-aid management of dental trauma in Iran: a basic premise for developing future intervention. Dent Traumatol. 2012;28(6):441-7.

08. de Franca RI, Traebert J, de Lacerda JT. Brazilian dentists' knowledge regarding immediate treatment of traumatic dental injuries. Dent Traumatol. 2007;23(5):287-90.

09. Frujeri Mde L, Costa ED, Jr. Effect of a single dental health education on the management of permanent avulsed teeth by different groups of professionals. Dent Traumatol. 2009;25(3):262-71.

10. Andersson L, Andreasen JO, Day P, Heithersay G, Trope M, Diangelis AJ, et al. International Association of Dental Traumatology guidelines for the management of traumatic dental injuries: 2. Avulsion of permanent teeth. Dent Traumatol. 2012;28(2):88-96.

11. Diangelis AJ, Andreasen JO, Ebeleseder KA, Kenny DJ, Trope M, Sigurdsson A, et al. International Association of Dental Traumatology guidelines for the management of traumatic dental injuries: 1 . Fractures and luxations of permanent teeth. Dent Traumatol. 2012;28(1):2-12

12. McIntyre JD, Lee JY, Trope M, Vann WF, Jr. Effectiveness of dental trauma education for elementary school staff. Dent Traumatol. 2008;24(2):146-50.

13. Wong FS, Kolokotsa K. The cost of treating children and adolescents with injuries to their permanent incisors at a dental hospital in the United Kingdom. Dent Traumatol. 2004;20(6):327-33.

14. Alves DF, Veloso HHP, Sayão S. O traumatismo dental e as repercussões endodônticas. In: Sayão $S$, editor. Endodontia Ciência tecnologia e arte: do diagnóstico ao acompanhamento. São Paulo: Santos; 2007. p. 209-33.

15. McIntyre JD, Lee JY, Trope M, Vann WF, Jr. Elementary school staff knowledge about dental injuries. Dent Traumatol. 2008;24(3):289-98.

16. Young C, Wong KY, Cheung LK. Emergency management of dental trauma: knowledge of Hong Kong primary and secondary school teachers. Hong Kong Med J. 2012;18(5):362-70.

17. Mesgarzadeh AH, Shahamfar M, Hefzollesan A. Evaluating knowledge and attitudes of elementary school teachers on emergency management of traumatic dental injuries: a study in an Iranian urban area. Oral Health Prev Dent. 2009;7(3):297-308.

18. Bayrak S, Tunc ES, Sari E. Evaluation of elementary school teachers' knowledge and attitudes about immediate emergency management of traumatic dental injuries. Oral Health Prev Dent. 2012;10(3):253-8.

19. Haragushiku GA, Faria MI, da Silva SR, Gonzaga CC, Baratto-Filho F. Knowledge and attitudes toward dental avulsion of public and private elementary schoolteachers. J Dent Child. 2010;77(1):49-53.

20. Al-Obaida M. Knowledge and management of traumatic dental injuries in a group of Saudi primary schools teachers. Dent Traumatol. 2010;26(4):338-41.

21. Arikan V, Sonmez H. Knowledge level of primary school teachers regarding traumatic dental injuries and their emergency management before and after receiving an informative leaflet. Dent Traumatol. 2012;28(2):101-7.

22. Vergotine RJ, Govoni R. Public school educator's knowledge of initial management of dental trauma. Dent Traumatol. 2010;26(2):133-6.

23. Al-Asfour A, Andersson L, Al-Jame Q. School teachers' knowledge of tooth avulsion and dental first aid before and after receiving information about avulsed teeth and replantation. Dent Traumatol. 2008;24(1):43-9.

24. Castilho LR, Sundefeld ML, de Andrade DF, Panzarini SR, Poi WR. Evaluation of sixth grade primary schoolchildren's knowledge about avulsion and dental reimplantation. Dent Traumatol. 2009;25(4):429-32.

25. Trope M. Avulsion of permanent teeth: theory to practice. Dent Traumatol. 2011;27(4):281-94.

26. Gopikrishna V, Baweja PS, Venkateshbabu N, Thomas T, Kandaswamy D. Comparison of coconut water, propolis, HBSS, and milk on PDL cell survival. J Endod. 2008;34(5):587-9.

\section{ABSTRACT}

Objective: To evaluate the knowledge of school teachers regarding the proper conduct in cases of dental trauma (DT) in school students. Material and methods: This study was conducted using a sample made up of 172 teachers from schools in João Pessoa-PB. These teachers answered a questionnaire concerning their knowledge about the emergency treatment of DTs. Results: Participants were mostly female $(79.6 \%)$ and were between 20 and 40 years old (49.4\%). Regarding the proper procedures to be taken in case of facial trauma, $78 \%$ said they would examine children's mouths. Regarding the possibility of saving a fractured tooth, $78.5 \%$ of respondents answered positively. In cases of avulsed teeth, it was found that $7.6 \%$ of teachers would not care about the tooth, only the child, and only $33.7 \%$ would look for the tooth. The ideal time to go to the dentist was reported by $52.8 \%$ as a maximum of 30 minutes. Regarding the storage medium, $8.1 \%$ would transport the tooth immersed in milk and $29.7 \%$ in saline. Conclusions: The teachers' knowledge concerning the most appropriate measure to be taken faced with TDs was inadequate. Further training of these professionals could minimize tooth loss.

Keywords: Knowledge; Emergencies; Tooth injuries. 


\section{AUTOR PARA CORRESPONDÊNCIA}

Heloísa Helena Pinho Veloso

Rua Vicente Barbosa dos Santos, 201, Apt. 201, Jardim

Oceânia, João Pessoa, PB, CEP.: 58037-445.

E-mail: hhveloso@gmail.com 